\title{
Numerical Simulation of Carbon Nanotubes/GaAs Hybrid PV Devices with AMPS-1D
}

\author{
Georgi Xosrovashvili ${ }^{1}$ and Nima E. Gorji ${ }^{2}$ \\ ${ }^{1}$ Department of Engineering, Ilia Chavchavadze State University, Kutaisi, 4600 Tbilisi, Georgia \\ ${ }^{2}$ Department of New Technologies, University of Tabriz, Tabriz 51566, Iran \\ Correspondence should be addressed to Nima E. Gorji; nima.s.gorji@gmail.com
}

Received 10 October 2013; Revised 9 December 2013; Accepted 25 December 2013; Published 27 March 2014

Academic Editor: Leonardo Palmisano

Copyright ( 2014 G. Xosrovashvili and N. E. Gorji. This is an open access article distributed under the Creative Commons Attribution License, which permits unrestricted use, distribution, and reproduction in any medium, provided the original work is properly cited.

\begin{abstract}
The performance and characteristics of a hybrid heterojunction single-walled carbon nanotube and GaAs solar cell are modelled and numerically simulated using AMPS-1D device simulation tool. The device physics and performance with different junction parameters are analysed. The results suggest that the open-circuit voltage changes very slightly by changing the electron affinity, acceptor and donor density while the other electrical parameters reach an optimum value. Increasing the concentration of a discrete defect density in the absorber layer decreases the electrical parameters. The current-voltage characteristics, quantum efficiency, band gap, and thickness variation of the photovoltaic response will be quantitatively considered.
\end{abstract}

\section{Introduction}

The unique electronic properties of single-walled carbon nanotubes (SWCNTs) are intriguing for many research groups in the present semiconductor technology to incorporate CNTs into CNT/III-V semiconductor hybrid devices $[1,2]$. On the other hand, GaAs has the superior electrical and optical properties owing to its diffusion length, welldeveloped industrial application compared with $\mathrm{Si}$, and some better electrical performance in certain aspects like higher carrier mobility [3]. Thus, from the energy conversion point of view, CNT/GaAs hybrid devices could be a promising optoelectronic combination since both materials are direct band gap semiconductors [4]. The solar cell constructed from SWCNT/GaAs thin film could efficiently absorb light energy with a wide absorption spectrum, if the diameter-dependent bandgap of the SWCNT was controlled through growth conditions from 0 to $2 \mathrm{eV}[5,6]$. Albeit the diode behaviour and photovoltaic response of the $\mathrm{CNT} / \mathrm{GaAs}$ heterojunction interface have been studied, the device modelling and simulations are still under investigation. In this work, for the first time, we used the one-dimensional simulation program called Analysis of Microelectronic and Photonic Structures
(AMPS-1D) device simulation tool for the modelling of the characteristics and performance parameters of a zigzag SWCNT/GaAs heterojunction solar cell. To our knowledge this is the first time that a hybrid heterojunction including a nanostructure material is going to be simulated in AMPS simulator or even in a simulator platform. This tool is widely used for the simulation of the thin film or silicon based photovoltaics [7-9].

\section{Device Model}

There are several methods to bring in contact the SWCNT absorber and GaAs (n-type substrate): chemical vapour deposition [10], followed by electron-beam lithography with assistance of a third material, that is, $\mathrm{Si}_{3} \mathrm{~N}_{4}$ or $\mathrm{SiO}_{2}$ [4].

A typical solar cell structure has been shown in Figure 1 where the $\mathrm{Au} / \mathrm{Pd}$ contact, $\mathrm{CNT}$ layer, n-type GaAs substrate, and $\mathrm{Au} / \mathrm{Pd}$ came in contact. Here we ignore any additional layer (e.g., $\mathrm{SiC}$ ) which in practice is deposited on the GaAs layer to cap the CNT layer. To proceed with the simulation, the material parameters employed as the inputs are selected based on the reported literature values or constrained to 


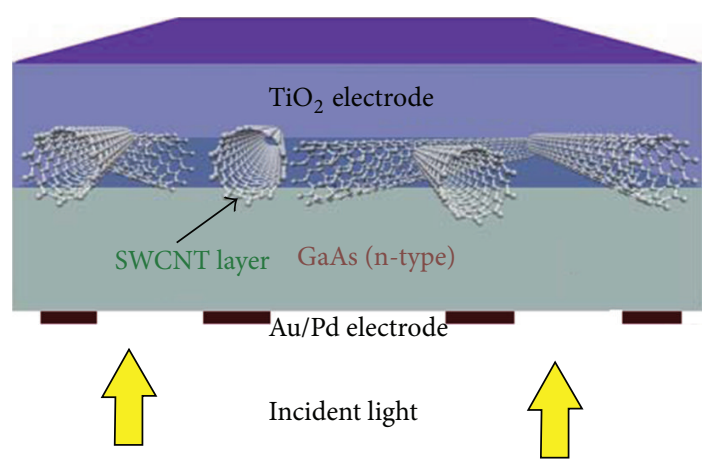

FIGURE 1: The heterojunction structure of the hybrid SWCNT/GaAs solar cell.

TABLE 1: The input parameters of AMPS-1D.

\begin{tabular}{lcc}
\hline Layer properties & SWCNT & GaAs \\
\hline Thickness $(\mathrm{nm})$ & 300 & 3000 \\
$\varepsilon / \varepsilon_{0}$ & 3.4 & 12.85 \\
$\mu_{e}\left(\mathrm{~cm}^{2} / \mathrm{v} \cdot \mathrm{s}\right)$ & $8 \times 10^{4}$ & 3917 \\
$\mu_{h}\left(\mathrm{~cm}^{2} / \mathrm{v} \cdot \mathrm{s}\right)$ & $2 \times 10^{3}$ & 239 \\
$N_{d}\left(\mathrm{~cm}^{-3}\right)$ & - & $2 \times 10^{15}$ \\
$N_{A}\left(\mathrm{~cm}^{-3}\right)$ & $4 \times 10^{14}$ & - \\
$N_{c}\left(\mathrm{~cm}^{-3}\right)$ & $5 \times 10^{16}$ & $4 \times 10^{18}$ \\
$N_{v}\left(\mathrm{~cm}^{-3}\right)$ & $6 \times 10^{17}$ & $8 \times 10^{17}$ \\
$E_{g}(\mathrm{ev})$ & 1.10 & 1.42 \\
$\chi(\mathrm{ev})$ & 4.27 & 4.07 \\
\hline
\end{tabular}

$\mu_{e, h}$ : electron and hole mobility; $n, p$ : donor and acceptor density; $N_{c, v}$ : effective density of states in $\mathrm{CB}$ and $\mathrm{V} \mathrm{B} ; E_{g}$ : bandgap; $\chi$ : materials electron affinity; $\varepsilon, \varepsilon_{0}$ : the dielectric constants of the material and air.

reasonable ranges. The key semiconductor properties of the layers as the input parameters for the simulations are given in Table 1. Although no attempt was made to match the simulation results with the experimental data, the purpose is to analyse the performance parameters of the cell versus the material parameters.

\section{Simulation Results and Discussion}

The schematic energy-band diagram of a typical hybrid SWCNT/GaAs solar cell has been illustrated in Figure 2. Based on this band diagram, the current-voltage characteristics of this structure have been calculated in the dark and light conditions under one sun at AM1.5 standard conditions (Figure 3 ) in order to show electrical behaviour of the proposed structure in different operation conditions. The dependence of SWCNT's acceptor concentration $(p)$ on the performance parameters of the cell has been investigated. In the simulations, shown in Figure 4, obviously there is an optimum value for the $p$ acceptor concentration of about $10^{16} \mathrm{~cm}^{-3}$ that has a better performance than those with low doping concentration. Increasing the $p$ concentration increases the open circuit voltage, $V_{\mathrm{oc}}$, negligibly where it stays almost constant. However, the short circuit current, $J_{\mathrm{sc}}$,

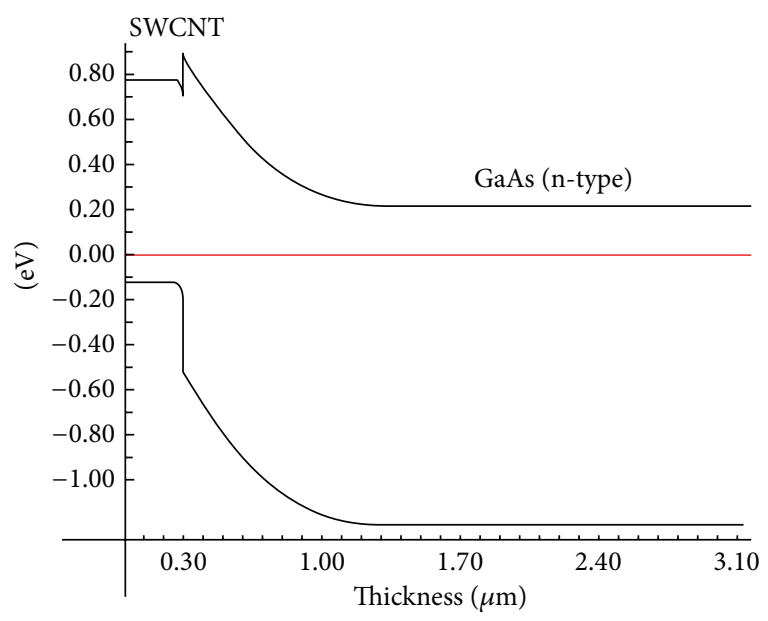

FIgURE 2: The energy-band diagram of a typical SWCNT/GaAs solar cell under equilibrium condition. The SWCNT layer has been extended to show better the band diagram.

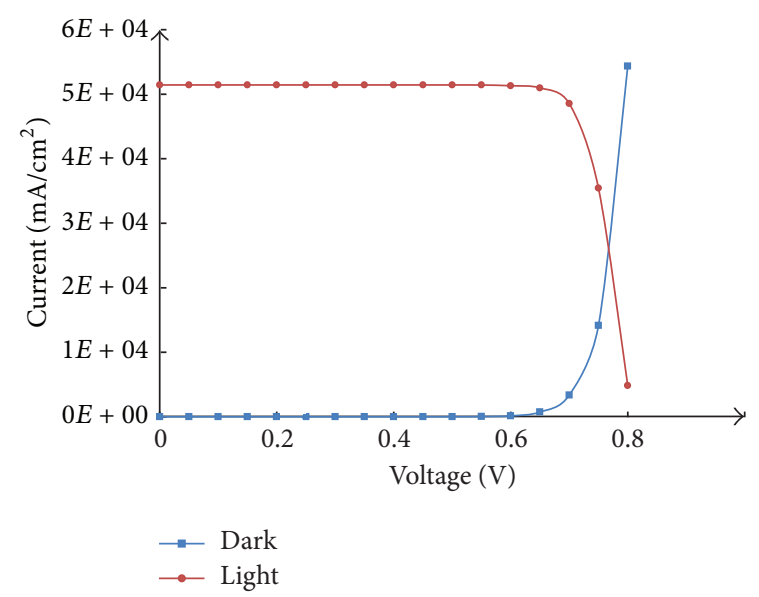

FIgURE 3: The current-voltage characteristics calculated by AMPS in dark and under 1 sun standard conditions.

decreases and the energy conversion efficiency reduces. On the other hand, donor concentration in the n-type GaAs has the same role with optimum value of about $10^{16} \mathrm{~cm}^{-3}$ and after that while lower than this value the $V_{\text {oc }}$ decreases.

In contrast, the $J_{s c}$ increases very slightly by $N_{D}$, but the efficiency and $F F$ decrease as shown in Figure 5. Higher donor concentration excites more electrons to the conduction band while higher acceptor concentration provides more holes to the valence band which creates better conductivity and photocurrent generation leading to increased efficiency. We need to note that the concentration of the acceptor and donor in the absorber layer is very effective on the performance parameters of the cell since the ratio of $N_{A} / N_{D}$ changes the Fermi levels of electron and holes, respectively. This is due to the fact that the positive charge density in the SWCNT absorber layer is higher than in the window layer. The $N_{A}$ increase at the junction of the window and absorber layer has no influence on the Fermi levels of 


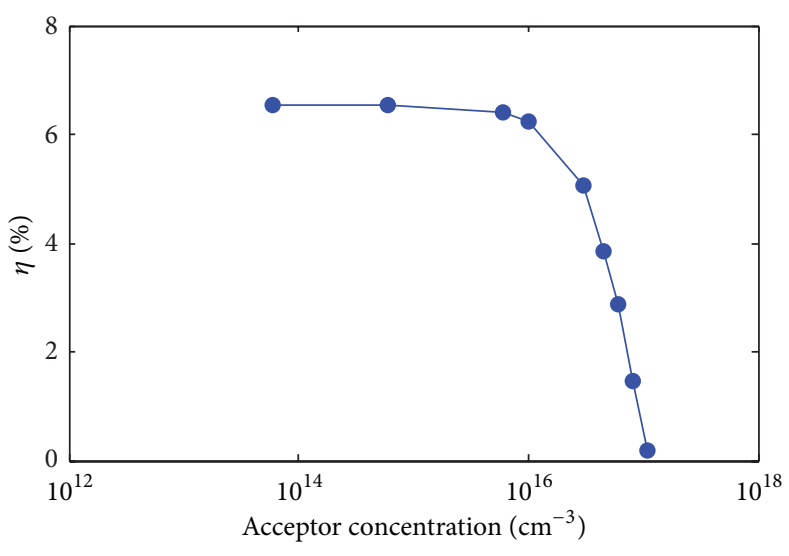

(a)

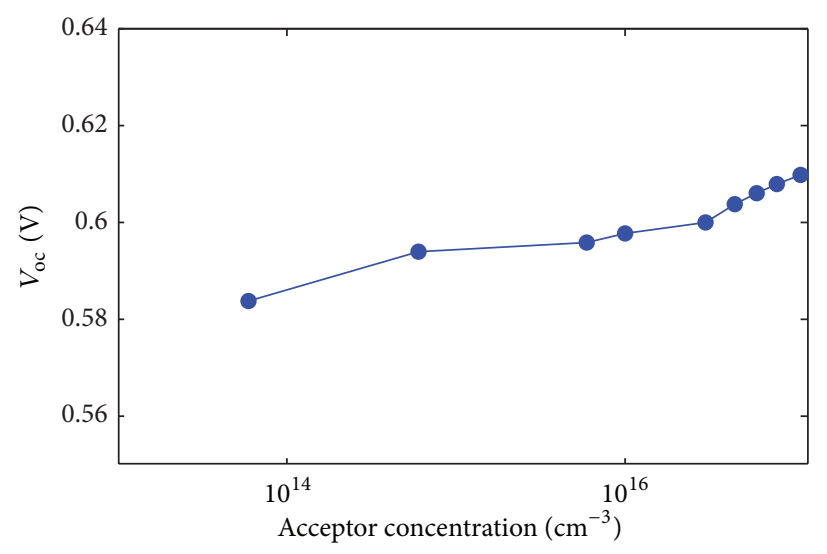

(c)

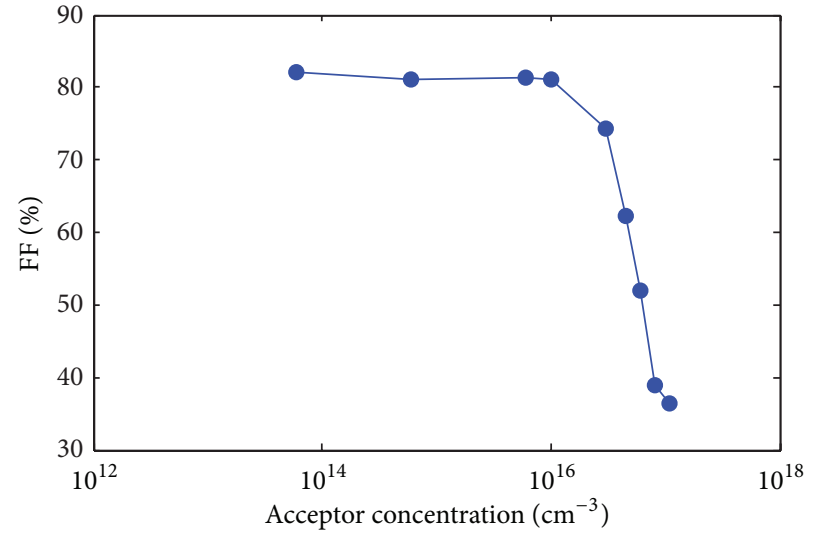

(b)

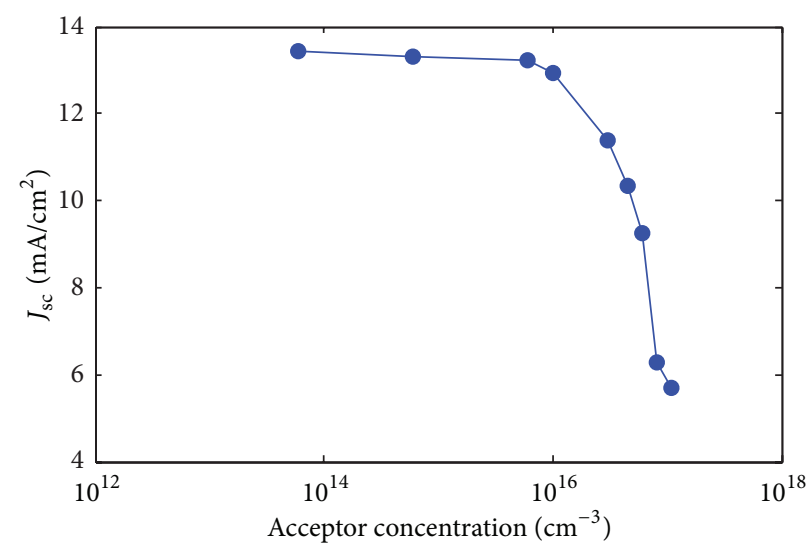

(d)

FIGURE 4: The effect of acceptor concentration, $(p)$ in the SWCNT layer, on the performance parameters with an optimum value of about $10^{16} \mathrm{~cm}^{-3}$.

the absorber layer because the increase in the positive charges (holes) is screened by electrons in an accumulation region of the window layer at the space charge region.

In Figure 6, we considered the variation in the electron affinity of both semiconductor layers as well. The electron affinity, $\chi$, is the minimum energy needed to move an electron from the Fermi level into vacuum level. There is a very small increase in $V_{\text {oc }}$ with the increased $\chi$ of SWCNT but a slight decrease in $V_{o c}$ of the GaAs. However, in both cases, the performance parameters increase to an optimum value by increasing the $\chi$. We also considered the effect of the bandgap of each layer on the performance parameters. The efficiency and $F F$ decrease after the optimum interval of 1.10 and $1.24 \mathrm{eV}$ for SWCNT and GaAs layer, respectively. In this regard, in Figure 7 the simulated external quantum efficiency (QE) is presented for 1 sun illumination condition.

It is apparent that the drop of efficiency after $500 \mathrm{~nm}$ is associated with the fall of the FF. The enhancement in the short wavelength can be due to the improvement of the $J_{\mathrm{sc}}$ which is obtained for lower $p$ and higher $n$ concentration levels. This is because the carrier collection will improve by additional electrons. In the SWCNT absorber layer as p-type, the electrons are the minority carriers and the transportation of this minority might be improved by doping to a proper level. Further consideration on the thickness of the layers gives the optimum values for the electrical parameters at the given values of the thickness in Table 1. The cell performance also varies with the operating temperature. For higher temperatures than $300 \mathrm{~K}$, the cell showed lower stability with lower $V_{\mathrm{oc}}$ and performance (not shown here). Once we have optimal values for doping concentrations and thickness of the layers (summarized in Table 1), we can simulate the solar cell structure shown in Figure 2. The semiconductors of our heterostructures may contain defect states within the band gap. If the defect states become charged, they add to the space charge of the heterostructures, similar as interface states. Thus, they influence the potential drop in the layers. Certainly, defect states in the band gap also give rise to carrier recombination. This is the reason why the $V_{\text {oc }}$ reduces. In the case that there is more than one deep defect level in the band gap, the recombination rates are added up as well as their contributions to the current densities. However, within the three-dimensional absorber (i.e., SWCNT) there may be distinct zones of particularly high defect densities: grain boundaries and regions of high strain, accumulated 


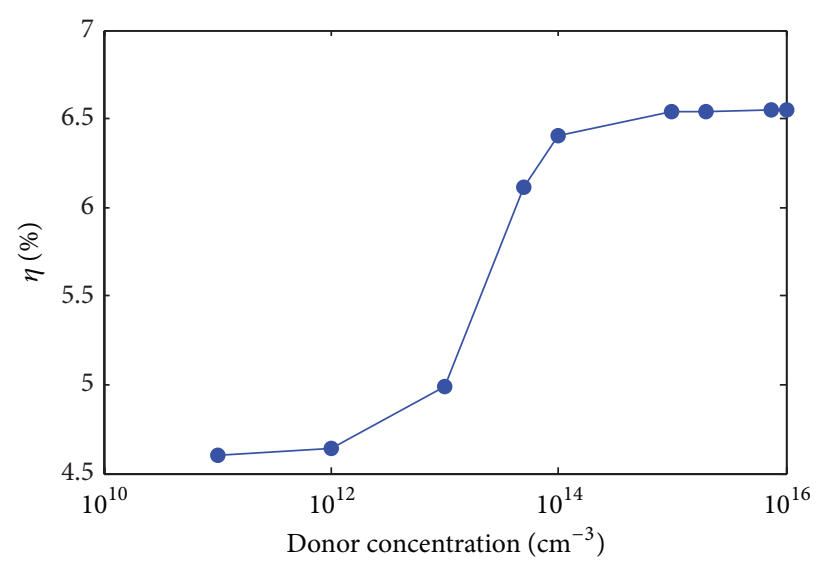

(a)

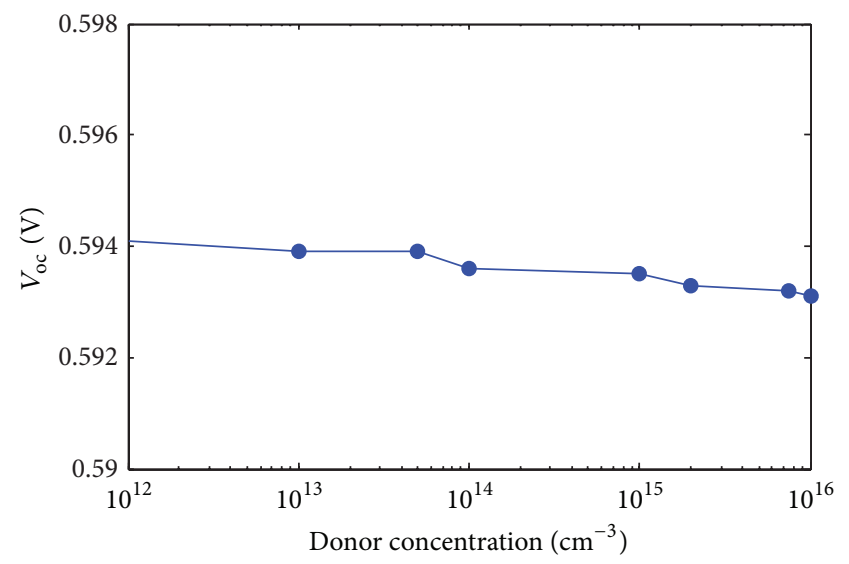

(c)

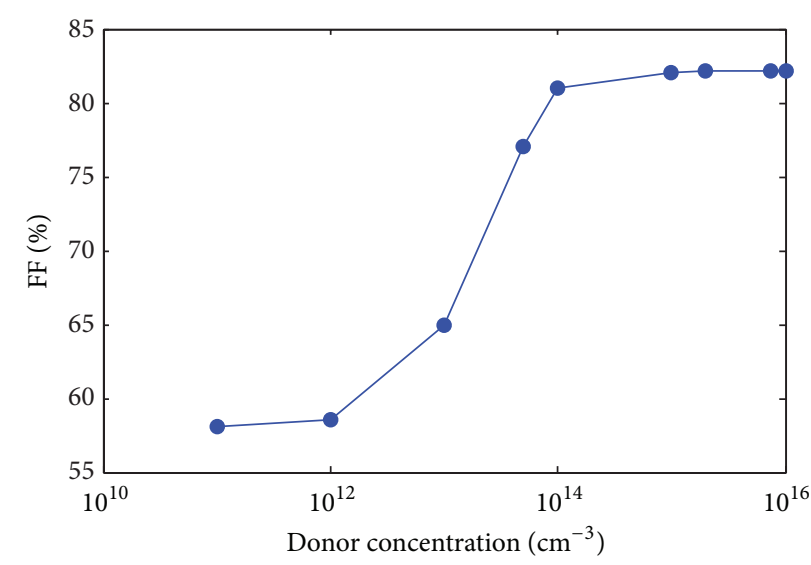

(b)

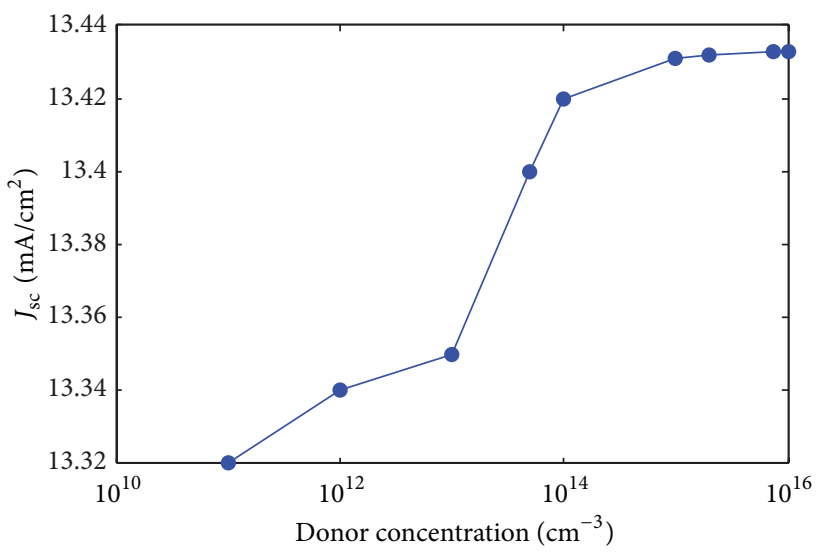

(d)

Figure 5: The effect of donor concentration, $(n)$ in the GaAs n-type layer, on the performance parameters with an optimum value of about $10^{16} \mathrm{~cm}^{-3}$.

impurities, or stoichiometry deviations. The performance of the solar cells is influenced by the quality of interfaces and formation of defects such as point defects, stacking faults, twins, dislocations, and grain boundaries. It is important to understand the defect physics so that appropriate methods may be developed to suppress the formation of harmful defects. This is the reason why the performance in Figure 8 is reducing by increased defect concentration.

Further considerations on the critical issues of this structure can be done to attain the high-efficiency heterojunction. For example, the effects of interface properties in the surface and interface defect layer on the performance parameter can be achieved. The simulation investigation along with the practical work on the growth and the preventing of damage to the SWCNT/GaAs wafer needs to be done in order to optimize the cell performance. Further consideration by AMPS tool can be done for the nanostructured solar cells.

For example nanoparticle solar cells can be modelled in this platform by adding a number of quantum dot layers in the active region and assuming the nanoparticles as defects which can be defined in this tool. Note that in these simulations we assumed a defect-free device to simplify the results. However, the more detailed studies can be done elsewhere.

\section{Summary}

A hybrid heterojunction solar cell based on carbon nanotube/GaAs has been proposed and simulated using the onedimensional simulation program called Analysis of Microelectronic and Photonic Structures (AMPS-1D). The SWCNT layer was added as the absorber layer and the window layer was taken from GaAs. The value of the parameters was taken from the experimental results of the papers published about this heterojunction. The results well simulate the band diagram and the effect of the thickness and carrier concentration on the performance parameters of the cell. The effects of acceptor and donor concentration, electron affinity, band gap, and thickness of the layers as well as operating temperature on the cell performance parameters have been investigated with a view to find a more efficient 


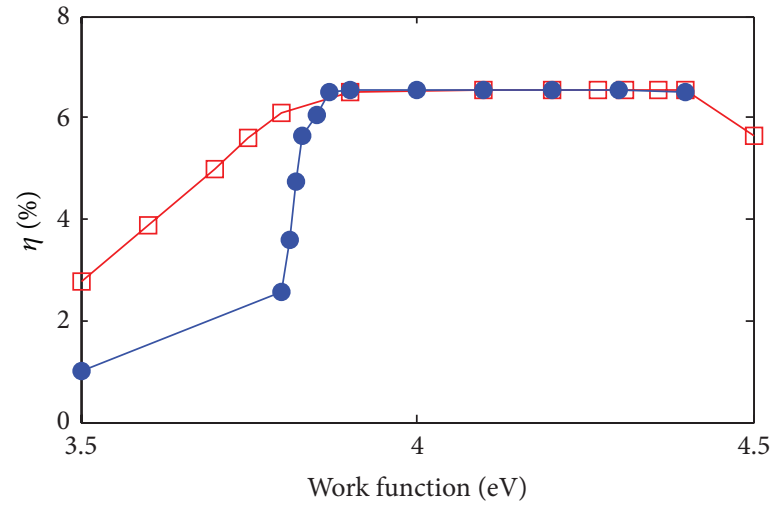

(a)

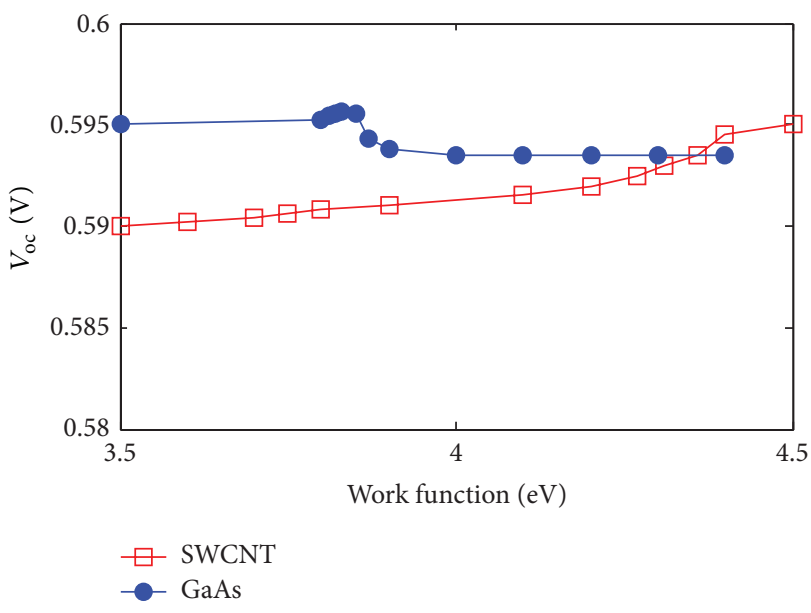

(c)

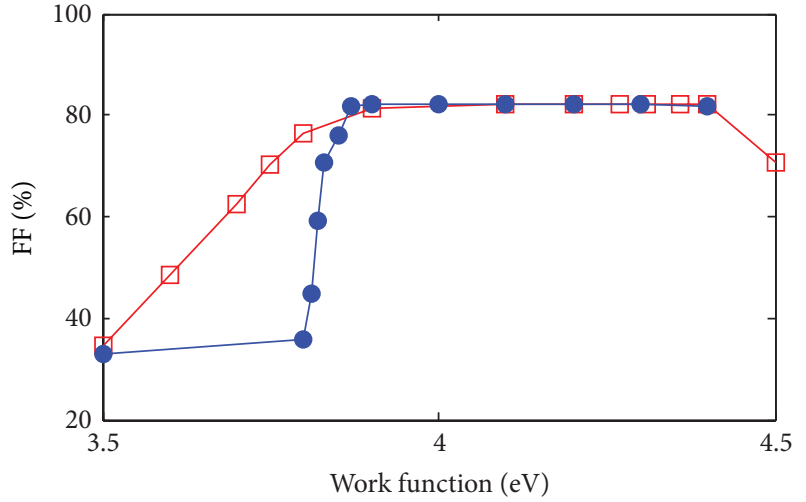

(b)

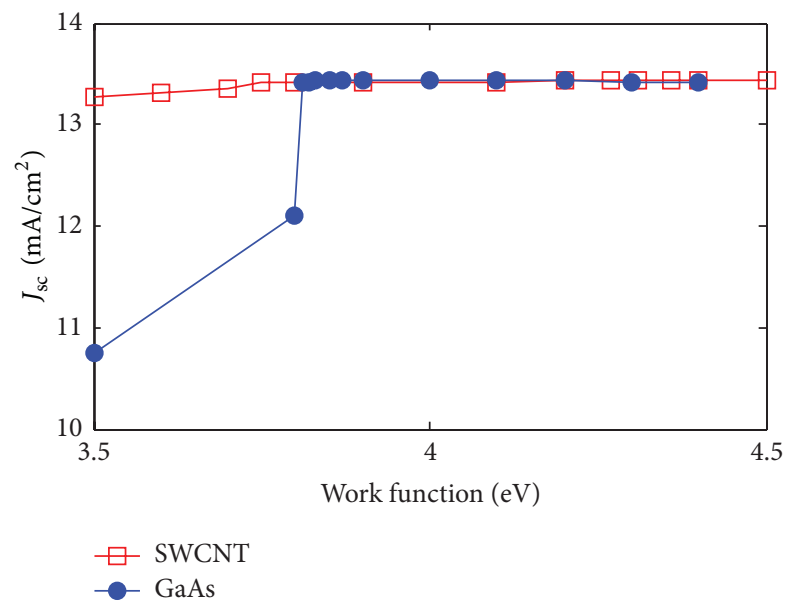

(d)

FIgURE 6: The effect of electron affinity $(\chi)$ on the performance parameters.

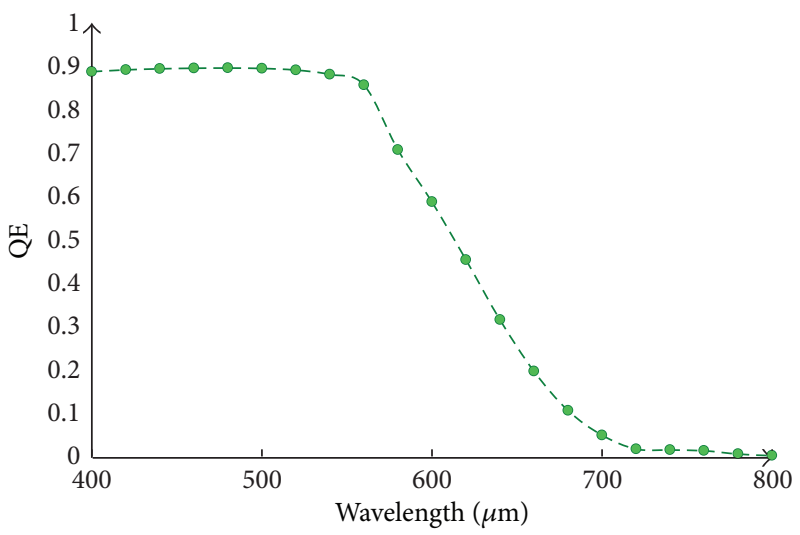

FIGURE 7: Quantum efficiency under one sun standard conditions.

cell. For this heterojunction, the maximum efficiency of $6.55 \%$ was achieved for a $300 \mathrm{~nm}$ and $3 \mu \mathrm{m}$ thickness of nanotube and GaAs layer, which decreases for the other values compared to the optimum values. The results are close to the reality since the parameter values were selected from the practical reported data in the literature. This work opens a road to explore in novel nanostructure solar cells configurations for the simulation of the nanostructured materials with AMPS getting a better view of the cell operation [11]. 

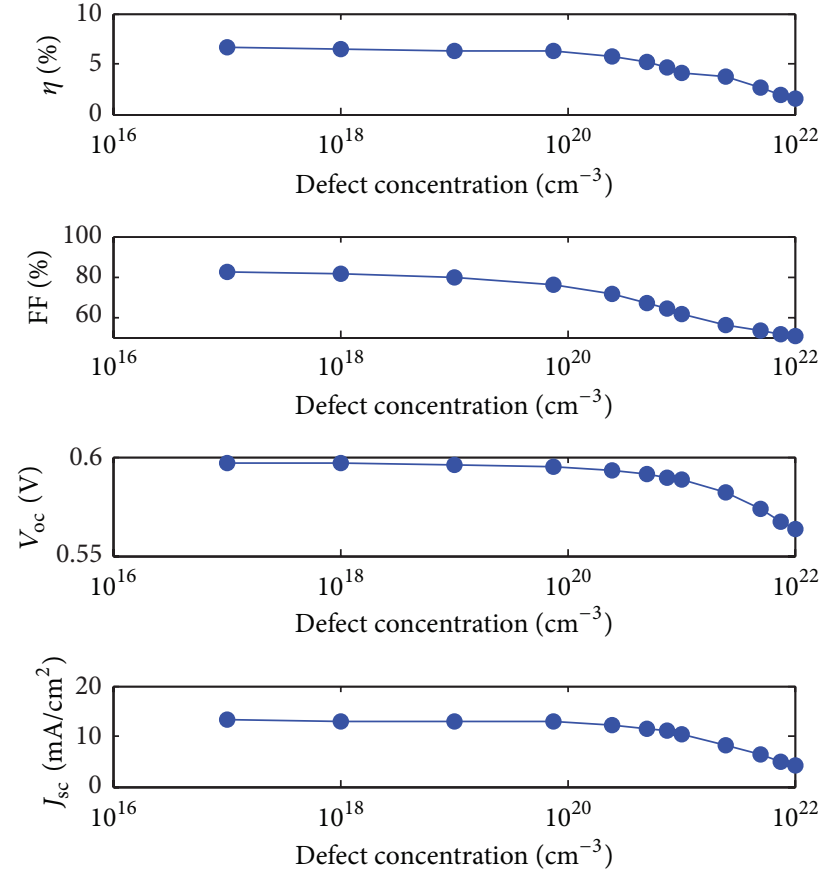

FIGURE 8: The impact of discrete defect concentration in the middle of the SWCNT layer band gap on the electrical parameters.

\section{Conflict of Interests}

The authors declare that there is no conflict of interests regarding the publication of this paper.

\section{Acknowledgments}

The authors gratefully acknowledges his support by a Senior Research Fellowship provided by the Nanotechnology Council of Iranian. The authors gratefully acknowledge Professor S. Fonash of the Pennsylvania State University (USA) and the Electric Power Research Institute for providing the AMPS-1D program used in the simulations.

\section{References}

[1] N. E. Gorji and M. Houshmand, "Carbon nanotubes application as buffer layer in $\mathrm{Cu}(\mathrm{In}, \mathrm{Ga}) \mathrm{Se}_{2}$ based thin film solar cells," Physica E: Low-Dimensional Systems and Nanostructures, vol. 50, pp. 122-125, 2013.

[2] D. Tune, F. Hennrich, S. Dehm et al., "The role of nanotubes in carbon nanotube-silicon solar cells," Advanced Energy Materials, vol. 3, no. 8, pp. 1091-1097, 2013.

[3] R. Engel-Herbert, Y. Takagaki, and T. Hesjedal, "Growth of carbon nanotubes on GaAs," Materials Letters, vol. 61, no. 2324, pp. 4631-4634, 2007.

[4] H. Li, W. Loke, Q. Zhang, and S. Yoon, "Physical device modeling of carbon nanotube/GaAs photovoltaic cells," Applied Physics Letters, vol. 96, no. 4, Article ID 043501, 3 pages, 2010.

[5] A. Behnam, J. Johnson, Y. Choi et al., "Metal-semiconductormetal photodetectors based on single-walled carbon nanotube
film-GaAs Schottky contacts," Journal of Applied Physics, vol. 103, no. 11, Article ID 114315, 6 pages, 2008.

[6] C. Liang and S. Roth, "Electrical and optical transport of GaAs/carbon nanotube heterojunctions," Nano Letters, vol. 8, no. 7, pp. 1809-1812, 2008.

[7] N. E. Gorji, U. Reggiani, and L. Sandrolini, "A modified equivalent circuit for CIGS solar cells," in Proceedings of the 27th European Photovoltaic Solar Energy Conference (EU PVSEC '12), pp. 2856-2859, Frankfurt, Germany, 2012.

[8] M. L. C. Cooray and V. G. Karpov, "Physical model of CdSbased thin-film photovoltaic junctions," Applied Physics Letters, vol. 88, no. 9, Article ID 093508, 3 pages, 2006.

[9] A. Belfar and H. Ait-Kaci, "Effect of incorporating p-type hydrogenated nanocrystalline silicon buffer layer on amorphous silicon n-i-p solar cell performances," Thin Solid Films, vol. 525, pp. 167-171, 2012.

[10] P. Ghosh, M. Subramanian, R. Afre et al., "Growth of Y-junction bamboo-shaped $\mathrm{CN}_{x}$ nanotubes on GaAs substrate using single feedstock," Applied Surface Science, vol. 255, no. 8, pp. 4611-4615, 2009.

[11] N. E. Gorji, U. Reggiani, and L. Sandrolini, "Graded band gap CIGS solar cells considering the valence band widening," in Proceedings of the 38th IEEE Photovoltaic Specialists Conference (PVSC '12), pp. 906-908, Austin, Tex, USA, June 2012. 

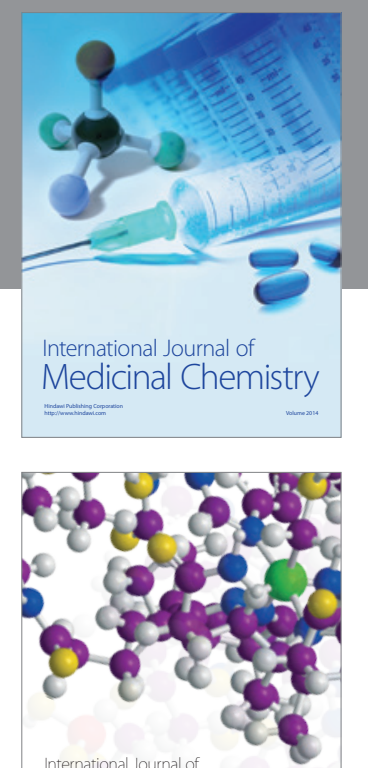

\section{Carbohydrate} Chemistry

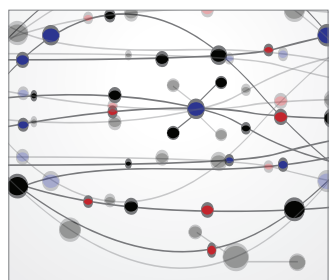

The Scientific World Journal
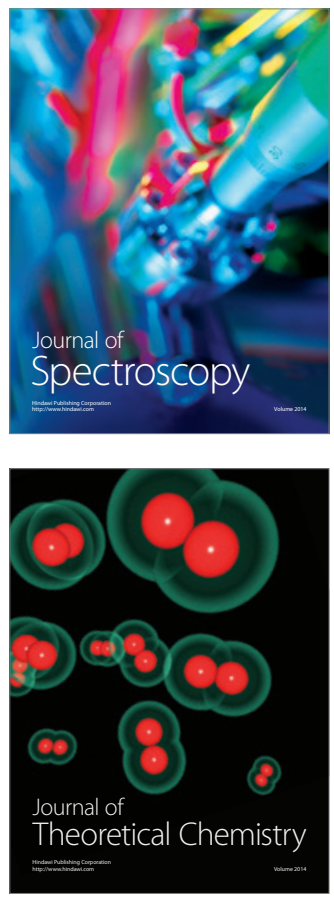
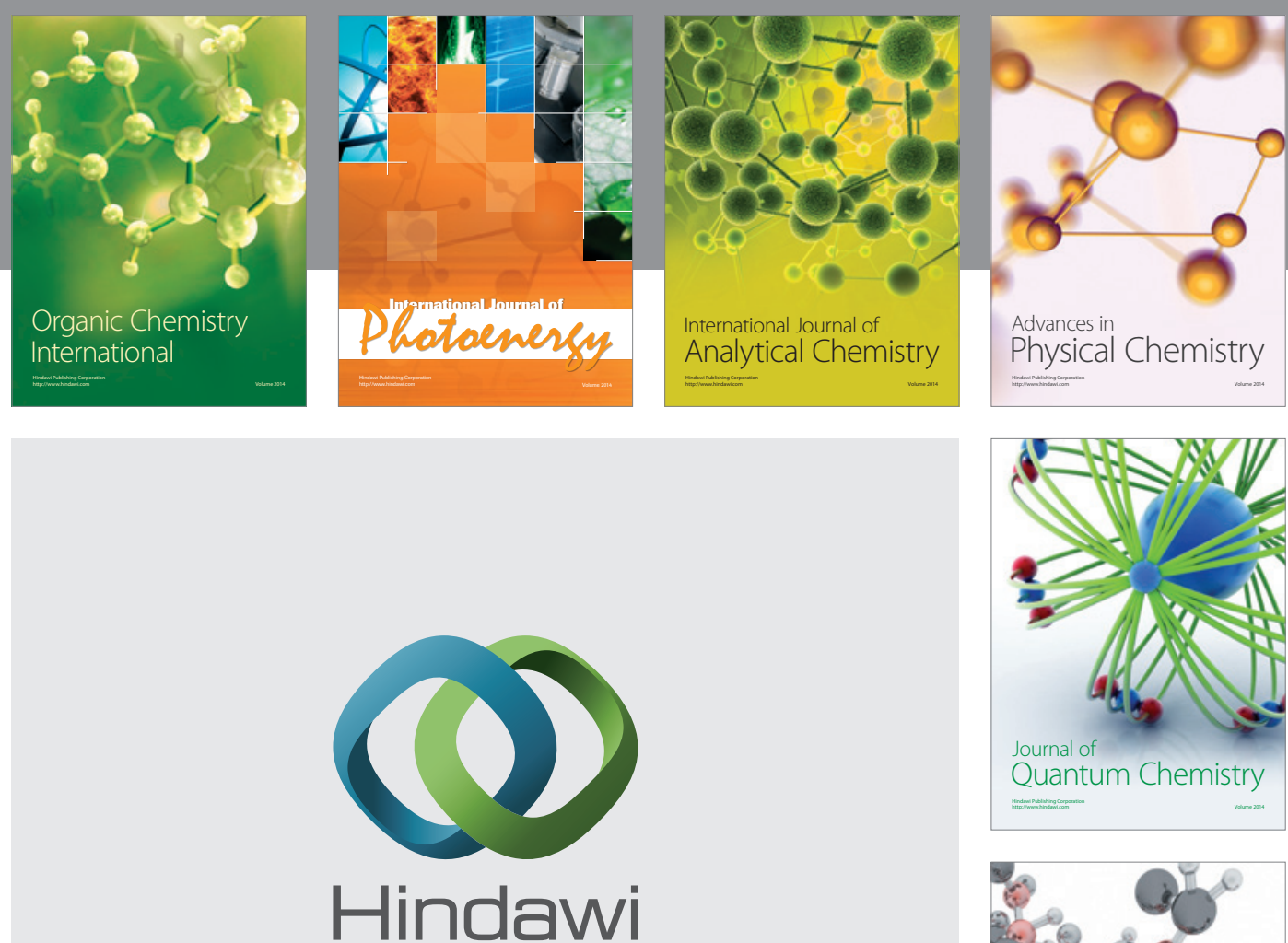

Submit your manuscripts at

http://www.hindawi.com

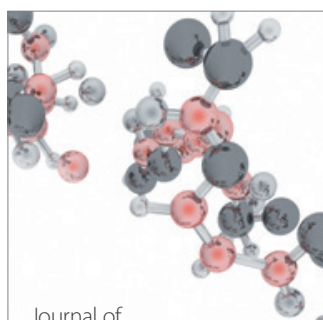

Analytical Methods

in Chemistry

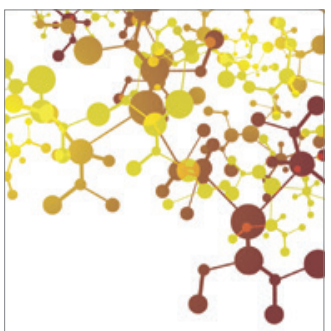

Journal of

Applied Chemistry

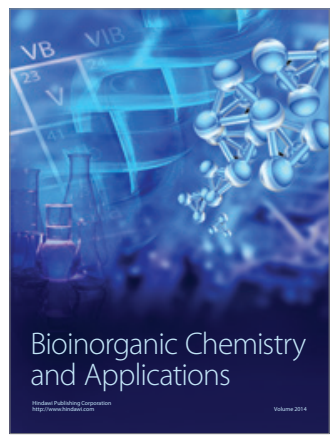

Inorganic Chemistry
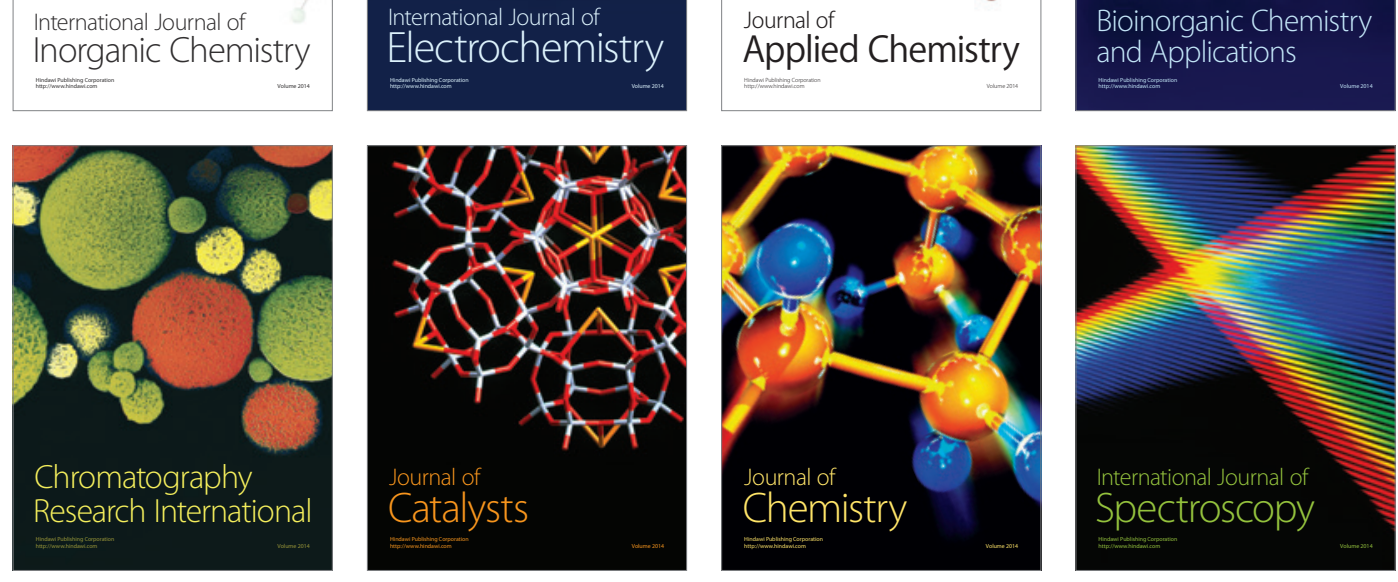